\title{
Of onions and men: Report of cavitary community acquired pneumonia due to Burkholderia cepacia complex in an immunocompetent patient and review of the literature
}

\author{
Ravi Manglani, Ester Sherman, Anna Shengelia, Oleg Epelbaum \\ Division of Pulmonary, Critical Care, and Sleep Medicine, Westchester Medical Center, Valhalla, NY, USA
}

\begin{abstract}
Burkholderia cepacia complex consists of highly antibiotic resistant gram negative bacilli that are plant symbionts and also potential agents of human infection. This bacterial family's claim to fame in clinical medicine is as the scourge of cystic fibrosis patients, in whom it is a notorious respiratory pathogen. Outside of cystic fibrosis, it rarely comes to mind as an etiology of community acquired pneumonia with or without lung cavitation in immunocompetent hosts. We describe a case of an otherwise healthy, community-dwelling man who presented with subacute
\end{abstract}

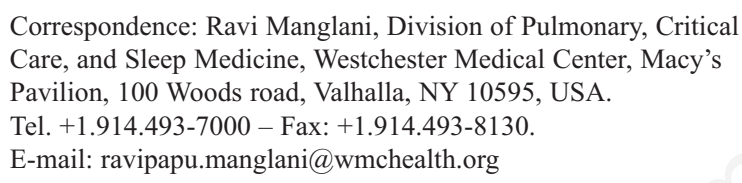

Key words: Burkholderia cepacia complex; cavitary pneumonia; immunocompetent.

Contributions: Each of the Authors participated in curation of literature, formulation of the manuscript from conception to design, analysis and finished product. Dr. Oleg Epelbaum played a supervisory role, as Senior Author, in the conception of the topic, editing, analysis, revision of intellectual content, and in literature review. The work has been reviewed and approved by all co- authors.

Conflict of interest: The Authors report no conflicts of interest.

Funding: The Authors do not report the receipt of any form of funding for the project.

Ethics approval: No ethical committee approval was required for this case report by the Department, because this article does not contain any studies with human participants or animals.

Received for publication: 2 July 2020.

Accepted for publication: 8 July 2020.

${ }^{\circ}$ Copyright: the Author(s), 2020

Licensee PAGEPress, Italy

Monaldi Archives for Chest Disease 2020; 90:1484

doi: 10.4081/monaldi.2020.1484

This article is distributed under the terms of the Creative Commons Attribution Noncommercial License (by-nc 4.0) which permits any noncommercial use, distribution, and reproduction in any medium, provided the original author(s) and source are credited. cavitary lung disease, the causative organism of which turned out to be Burkholderia cepacia complex. Our report is accompanied by a review of the literature, which identified an additional eleven cases in the same category. We analyze all of the available cases for the emergence of any identifiable patterns or peculiarities.

\section{Introduction}

Burkholderia cepacia and related species, known collectively as Burkholderia cepacia complex, is a well-established cause of morbidity and mortality in the cystic fibrosis (CF) population. However, as a cause of community acquired pneumonia it may be an emerging pathogen with a peculiar ecological niche in the plant biosphere. Herein, we present a case of cavitary pneumonia in an immunocompetent host without a history of CF that initially roused suspicion for mycobacterial or fungal infection, but ultimately turned out to be an example of CAP due to Burkholderia cepacia complex, which is an etiology of lung cavitation likely to be underappreciated by pulmonary clinicians. The infection poses special challenges due to its propensity for antibiotic resistance. The accompanying literature review indicates that this is the twelfth case of its kind reported in the English language but the first from the United States since the year 2000.

\section{Case Report}

A 71-year-old man presented to our institution, located in the suburbs of New York City with two weeks of productive cough with blood-streaked sputum, heavy night sweats, and anorexia. Before presentation, he had completed outpatient courses of azithromycin and cefuroxime with no improvement. His past medical history was limited to hypertension and hyperlipidemia. He had no personal history of tuberculosis nor any known exposures. He had never smoked and was an avid bicyclist with unlimited effort tolerance. He was originally from Chile, having immigrated to the United States 35 years previously and having last traveled back five years earlier. In Chile, he had worked as a food vendor, and in the United States he had been a factory machinist until retirement. On admission, he was afebrile with an oxygen saturation of $99 \%$ while breathing room air. Physical examination was significant only for bronchial breath sounds over the right chest anteriorly. Routine laboratory evaluation was remarkable for an elevated blood leukocyte count of $13.2 \mathrm{~K} / \mathrm{mm}^{3}$ (normal range $4.8-10.8 \mathrm{~K} / \mathrm{mm}^{3}$ ) with a neutrophil predominance $(84.5 \%)$. The serum procalcitonin level was $0.08 \mathrm{ng} / \mathrm{mL}$ (lower limit of normal $0.15 \mathrm{ng} / \mathrm{mL}$ ). Testing for the 
human immunodeficiency virus was negative. Chest radiography revealed a large thick walled cavity in the apex of the right lung (Figure 1A). Fortuitously, the patient had undergone a right shoulder $\mathrm{X}$-ray three weeks prior to this presentation, which captured the right lung, but did not demonstrate an apical lesion (Figure 1B). Subsequent computed tomography (CT) of the chest performed following administration of intravenous contrast showed multiple cavitary masses in the right apex (Figure 2) with associated right hilar lymphadenopathy (not shown). Upon further questioning, the patient denied recent dental procedures and any reported episodes of syncope. At this point, the main differential diagnosis for the cavitary lung process included malignancy and infection, the latter encompassing indolent bacterial pathogens as well as mycobacteria and fungi. The absence of this abnormality on a radiograph obtained three weeks earlier suggested a rapid tempo of evolution, rendering malignancy and tuberculosis (TB) less likely possibilities. Further serological evaluation yielded negativity for $1,3-\beta$-D-glucan; galactomannan; Cryptococcus and Histoplasma antigen; Coccidioides, Blastomyces, and Histoplasma antibodies. After serial sputum smears returned negative for acid-fast bacilli, the patient underwent bronchoscopic evaluation for the causative agent. Endobronchial ultrasound-guided transbronchial needle aspiration samples from lymph node stations 7 and 11R were non-diagnostic. Cytology specimens from fine needle aspiration of the cavitary lesions as well as from bronchoalveolar lavage (BAL) fluid contained only neutrophils and macrophages. Transbronchial biopsy tissue showed chronic inflammation. Four days following the procedure, bacterial culture of BAL fluid and biopsy tissue grew a gram negative bacillus identified as Burkholderia cepacia complex (BCC) by Matrix Assisted Laser Desorption Ionization-Time of Flight Mass Spectrometry (MALDI/TOF). To exclude confusion with similar bacterial pathogens such as Pseudomonas species, identification was additionally confirmed by $16 \mathrm{~S}$ ribosomal sequencing. The patient was discharged on this trimethoprim/sulfamethoxazole, in keeping with the sensitivities of the isolate. He denied a history of recurrent respiratory tract infections. Repeat CT chest performed after six weeks of therapy demonstrated marked interval improvement of the cavitary pneumonia (Figure 3).

\section{Discussion}

Burkholderia is a genus of gram negative, non-lactose fermenting aerobic bacilli that includes $B$. cepacia and many other genetically related species that are collectively referred to as BCC. Prior to 1992, these bacteria were classified under the genus Pseudomonas, but were transferred to their current genus based on distinct molecular genetics [1]. Clinically, differentiation of BCC from Pseudomonas spp is vital because it is a notoriously resistant organism, including to antipseudomonal antibiotics. The genus Burkholderia is named after William Burkholder, who identified these bacteria in 1950 as the cause of rotting onion bulbs [2]. Since then, it has been recognized that BCC is a ubiquitous plant colonizer and is vital to crop preservation due to its pesticidal properties [3]. Some, but not all, members of BCC are pathogenic to humans, and their natural habitat in the plant environment is believed to be the reservoir for human infection. BCC is uniquely capable of surviving in nutrient-poor liquid media, colonization of which in hospital settings has resulted in outbreaks due to contaminated mouthwash [4], albuterol nebulization solution [5], injection fluids [6], liquid and intravenous medications $[7,8]$ and chemical detergents [9].
$\mathrm{BCC}$ is associated with three main categories of human infection of importance to the pulmonary and critical care community. The first is nosocomial bacteremia, typically in intensive care unit settings, which is believed to result from transmission via invasive devices such as central venous catheters and bronchoscopes [10]. The second, and the one for which BCC is particularly well known,

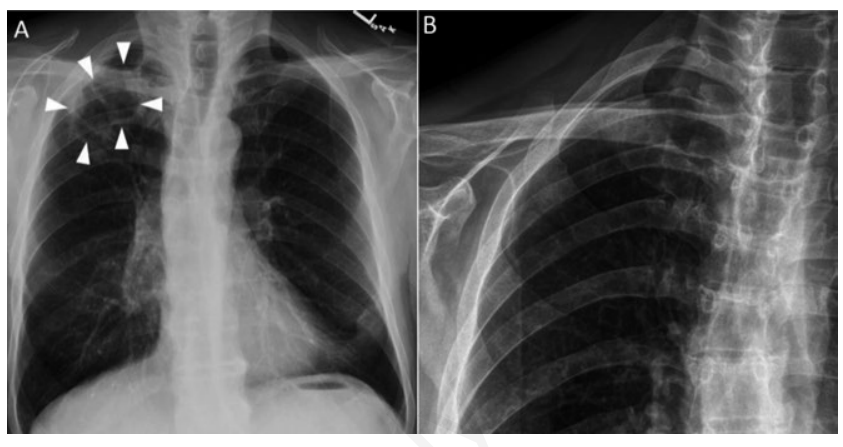

Figure 1. A) Frontal chest radiograph showing a cavitary lesion in the right lung apex (arrowheads). B) Right shoulder radiograph obtained three weeks before the chest radiograph in panel A demonstrates the absence of a right apical lesion at that time.

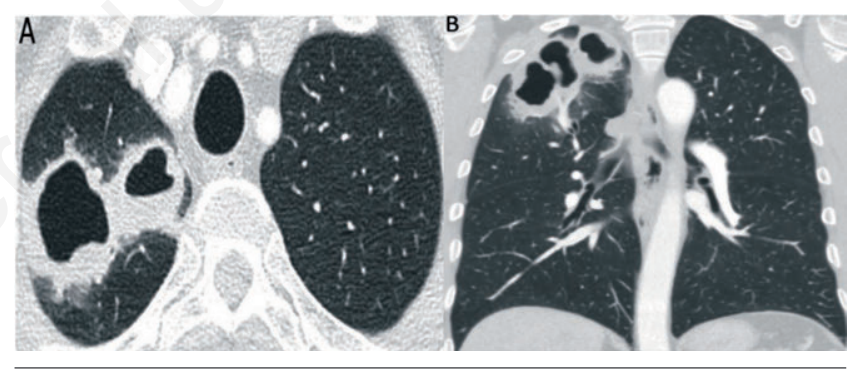

Figure 2. A) Chest computed tomography axial section set to lung window demonstrating a large, thick-walled, multi-cavitary lesion in the apical segment of the right upper lobe. B) Coronal reconstruction image from the same study as panel $A$ highlights the extent and multi-cavitary nature of the lesion.

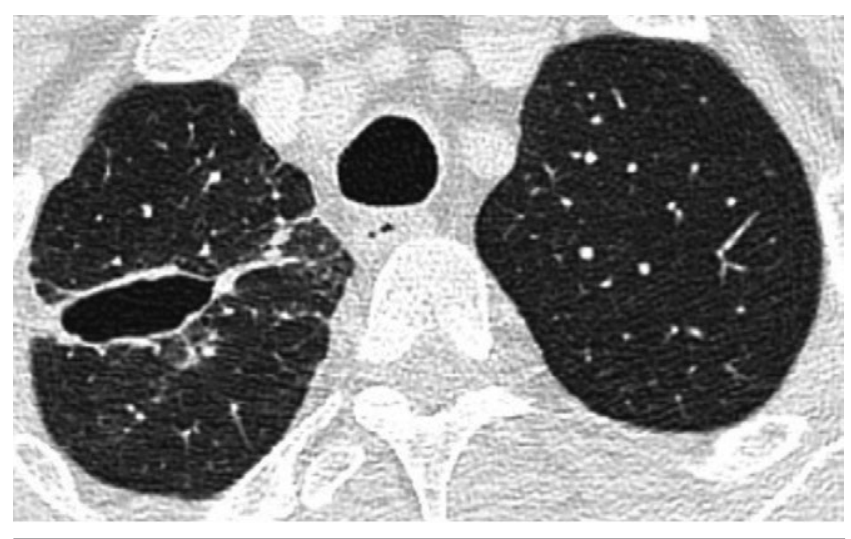

Figure 3. Chest computed tomography axial section set to lung window performed following six weeks of antibiotic therapy showing marked interval diminution in the size of the multi-cavitary right apical lesion. 
is lower respiratory tract infection in colonized patients with $\mathrm{CF}$. Although $B$. multivorans is the most common species in the $\mathrm{CF}$ population, $B$. cenocepacia is the most virulent and is associated with so-called "cepacia syndrome," a dreaded complication characterized by overwhelming pneumonia, bacteremia, and very high mortality [11]. In most centers, detection of B. cenocepacia in a CF patient precludes lung transplantation because of markedly inferior outcomes [12]. Another especially vulnerable host is the rare patient with chronic granulomatous disease (CGD) in which neutrophils are defective in oxidative burst-mediated elimination of phagocytosed microbes [13]. There was no evidence in our patient suggestive of either CF or CGD, and we are not privy to results of possible testing for these conditions during his ambulatory follow up. The third and rarest type of infection is community acquired pneumonia (CAP) caused by BCC in immunocompetent patients without documented or suspected CF. This category appears to encompass the present case and so was the subject of our literature review.

We performed a PubMed search using the terms "Burkholderia cepacia pneumonia" and manually reviewed the results to identify reports describing non-CF patients. We also searched Google Scholar with the same string to capture any publications not indexed in PubMed. The reference lists of pertinent articles identified through this strategy were screened for possible additional matches. Only English-language publications were extracted. Reports of non-CF BCC cases involving institutional [14] or nosocomial acquisition $[15,16]$, bacteremia without overt lung involvement [17], and transmission from CF patients to a family caregiver [18] were considered inapplicable. Our results are concordant with those of microbiology colleagues who recently likewise performed a literature review of non-CF BCC CAP cases [19]. Table 1 summarizes the most salient features of the 12 cases, including the current one, described in available English-language publications.

Five of the 12 patients are accounted for by two case series: three patients reported by Belchis et al. [20] from the United States and two by Bayram et al. [21] from Turkey. The other seven patients are contributed by individual case reports. The Belchis series includes the only two female patients; the remaining ten are males, which is a noteworthy predominance. It also accounts for the majority $(3 / 5)$ of patients who died. This series is uniquely valuable as it provides a glimpse into the histopathology of BCC pneumonia through a combination of antemortem and postmortem tissue examinations. Microscopically, this infection is characterized by necrotizing granulomatous inflammation accompanied by microabscesses. These findings are reminiscent of those seen in melioidosis, a lung infection caused by $B$. pseudomallei and prevalent in Southeast Asia [22].

Cavitation is explicitly demonstrated or could be inferred [23] in more than half of reports (5/9) that describe the thoracic imaging. In reports from TB-endemic countries such as Turkey and India, as in our case of an immigrant to the United States, a common theme is initial concern for TB. Among those patients for whom an occupational history is available, culinary and agricultural work are a frequent motif, supporting food handling as the inoculation route for $\mathrm{BCC}$ in the community. Of note, our patient as well as the patient reported by Bayram et al. [21] had only remote occupational exposure, raising the possibility that colonization with BCC could give rise to active infection at a future point even in normal, previously asymptomatic hosts. Neither a history of smoking nor underlying chronic lung disease is consistently seen; only the patient reported by Ishizuka [24] had structural lung disease in the form of a resected aspergilloma. Many patients, similar to ours, have no identifiable immune compromise. Patient \#4 (Table 1) in the Belchis series suffered two bouts of pneumonia before the age of 40 , a history concerning for undiagnosed immunodeficiency. Patient \#5 was receiving mild immunosuppression with chloroquine. Although labeled immunocompetent by the authors, the patient described by Datta et al. [19] is a poorly controlled diabetic of long duration. Uncontrolled diabetes mellitus is an established cause of neutrophil dysfunction, perhaps capable of facilitating invasive BCC infection in a manner analogous to CGD [25]. Patient \#1 [26] is notable as the only pediatric case and also as the only recipient of chronic antibiotic therapy, which could have promoted $\mathrm{BCC}$ infection through the application of selection pressure. This patient survived but only after a stay on mechanical ventilation. The other survivors recovered without the need for intensive care.

Table 1. Summary of the salient features of reported cases of community-acquired pneumonia due to Burkholderia cepacia complex.

\begin{tabular}{|c|c|c|c|c|c|c|c|c|c|}
\hline$\#$ & Source & Location & Age/Sex & Occupation & Smoking & PMH & Cavitation & Culture & Outcome \\
\hline 1 & Pujol [26] 1992 & Spain & $14 \mathrm{M}$ & None & $\mathrm{N}$ & Acne $^{a}$ & $\mathrm{~N}$ & BD, PF, TTNA & Survived $^{b}$ \\
\hline 2 & Waterer [27] 1999 & US & $32 \mathrm{M}$ & Cook & $\mathrm{N}$ & HTN & $\mathrm{N}$ & $\mathrm{BD}$ & Survived \\
\hline 3 & Belchis [20] 2000 & US & $44 \mathrm{M}$ & NR & NR & Histo & NR & Autopsy & Died \\
\hline 4 & Belchis [20] 2000 & US & $40 \mathrm{~F}$ & NR & Y & PNA $^{\mathrm{c}}$ & NR & OLB & Died \\
\hline 5 & Belchis [20] 2000 & US & $43 \mathrm{~F}$ & NR & $\mathrm{N}$ & $\mathrm{SLE}^{\mathrm{d}}$ & NR & OLB & Died \\
\hline 6 & Ishizuka [24] 2003 & Japan & $69 \mathrm{M}$ & NR & NR & $\mathrm{ASG}^{\mathrm{e}}$ & $\mathrm{N}$ & SputumAutopsy & Died \\
\hline 7 & Bayram [21] 2011 & Turkey & $60 \mathrm{M}$ & Office (R) & Y & None & Y & BAL & Survived \\
\hline 8 & Bayram [21] 2011 & Turkey & $66 \mathrm{M}$ & Farmer (R) & Y & None & $\mathrm{N}$ & BAL & Survived \\
\hline 9 & Karanth [23] 2012 & India & $32 \mathrm{M}$ & Farmer & $\mathrm{N}$ & None & $Y^{f}$ & SputumBAL & Survived \\
\hline 10 & Suresh [28] 2013 & India & $63 \mathrm{M}$ & Farmer & Y & None & Y & Sputum & Survived \\
\hline 11 & Datta [19] 2020 & India & $71 \mathrm{M}$ & Farmer & NR & $\mathrm{DM}^{\mathrm{g}}$ & Y & Sputum & Died \\
\hline 12 & Present case & US & $71 \mathrm{M}$ & Food vendorhFactory & $\mathrm{N}$ & HTN & Y & BAL TBB & Survived \\
\hline
\end{tabular}

ASG, aspergilloma; BAL, bronchoalveolar lavage; BD, blood, DM, diabetes mellitus; Histo, histoplasmosis; HTN, hypertension; NR, not reported; OLB, open lung biopsy; PF, pleural fluid; PNA, pneumonia; R, retired; SLE, systemic lupus erythematosus; TBB, transbronchial biopsy; TTNA, transthoracic needle aspiration. ${ }^{\text {PPatient }}$ was receiving multiple antibiotics and a synthetic retinoid for the diagnosis of acne conglobate; ${ }^{b}$ after a period of mechanical ventilation; ${ }^{c}$ patient had been diagnosed with pneumonia twice in her thirties; in one of the two episodes, the causative pathogen was identified as Salmonella paratyphi B; ${ }^{\mathrm{d}}$ patient was

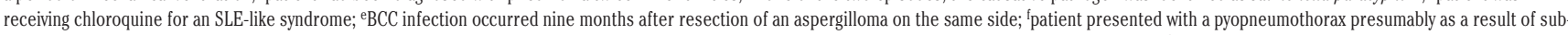
pleural cavity rupture, although computed tomography was not performed for confirmation; ${ }^{g}$ patient carried a diagnosis of long-standing poorly-controlled DM; ${ }^{\text {hin }}$ his home country of Chile, from which he had emigrated to the US 35 years earlier. 


\section{Conclusions}

$\mathrm{BCC}$, a resistant gram negative bacillus, warrants recognition as a potential cause of both cavitary and non-cavitary CAP in persons without known immune compromise and without pre-existing lung disease. As a colonizer of plant life, it may be an infection that accompanies certain geographic and occupational exposures. Consideration of BCC impacts antibiotic selection, and it will not be considered unless specifically suspected. The presence of lung cavitation without an obvious explanation ought to prompt inclusion of this rare but important agent of CAP on the list of possible culprits.

\section{References}

1. Mahenthiralingam E, Baldwin A, Dowson CG. Burkholderia cepacia complex bacteria: opportunistic pathogens with important natural biology. J Appl Microbiol 2008;104:1539-51.

2. Burkholder WH. Sour skin, a bacterial rot of onion bulbs. Phytopathology 1950;40:115-7.

3. Gautam V, Singhal L, Ray P. Burkholderia cepacia complex: beyond pseudomonas and acinetobacter. Indian $\mathrm{J}$ Med Microbiol 2011;29:4-12.

4. Dos Santos Saalfeld SM, Shinohara DR, Dos Anjos Szczerepa $\mathrm{MM}$, et al. Consecutive outbreaks of Burkholderia cepacia complex caused by intrinsically contaminated chlorhexidine mouthwashes. Am J Infect Control 2020. doi: 10.1016/j.ajic. 2020.04.025.

5. Hamill RJ, Cadle RM, Koza MA. Burkholderia cepacia and nebulized albuterol. Ann Inter Med 1996;124:274.

6. Brooks RB, Mitchell PK, Miller JR, et al. Multistate outbreak of Burkholderia cepacia complex bloodstream infections after exposure to contaminated saline flush syringes: United States, 2016-2017. Clin Infect Dis 2019;69:445-9.

7. Glowicz J, Crist M, Gould C, et al. A multistate investigation of healthcare-associated Burkholderia cepacia complex infections related to liquid docusate sodium contamination, January - October 2016. Am J Infect Control 2018;46:649-55.

8. Moehring RW, Lewis SS, Isaacs PJ, et al. Outbreak of bacteremia due to Burkholderia contaminans linked to intravenous fentanyl from an institutional compounding pharmacy. JAMA Intern Med 2014;174:606-12.

9. Lee CS, Lee HB, Cho YG, et al. Hospital-acquired Burkholderia cepacia infection related to contaminated benzalkonium chloride. J Hosp Infect 2008;68:280-2.

10. Bressler AM, Kaye KS, LiPuma JJ, et al. Risk factors for Burkholderia cepacia complex bacteremia among intensive care unit patients without cystic fibrosis: a case-control study. Infect Control Hosp Epidemiol 2007;28:951-8.

11. Dupont L. Lung transplantation in cystic fibrosis patients with difficult to treat lung infections. Curr Opin Pulm Med 2017;23:574-9.
12. Lobo LJ, Noone PG. Respiratory infections in patients with cystic fibrosis undergoing lung transplantation. Lancet Respir Med 2014;2:73-82.

13. Yu JE, Azar AE, Chong HJ, et al. Considerations in the diagnosis of chronic granulomatous disease. J Pediatric Infect Dis Soc 2018;7:s6-11.

14. Mohamed AM, Waheed SA, Mohamed NM, Clark NM. An 87year-old man with acute catastrophic pneumonia. Ann Am Thorac Soc2016;13:432-7.

15. Hauser N, Orsini J. Cepacia syndrome in a non-cystic fibrosis patient. Case Rep Infect Dis 2015;2015:537627.

16. Lim BA, Lopez A, Buensalido JA. Refractory Burkholderia cepacia bacteraemia from a consolidation pneumonia lasting more than 7 weeks, successfully treated with systemic antibiotics and nebulised meropenem. BMJ Case Rep 2019;12: e229566.

17. Ranjan R, Chowdhary P, Kamra A. Community acquired Burkholderia cepacia bacteraemia presenting as MODS in an immunocompetent individual: An unusual case. J Clin Diagn Res 2017;11:DD01-2.

18. Ledson MJ, Gallagher MJ, Walshaw MJ. Chronic Burkholderia cepacia bronchiectasis in a non-cystic fibrosis individual. Thorax 1998;53:430-2.

19. Datta P, Gupta M, Kumar MB, et al. Burkholderia cepacia complex causing pneumonia in an immunocompetent non-cystic fibrosis patient: Case report and review of literature. Infect Disord Drug Targets 2020;20:106-10.

20. Belchis DA, Simpson E, Colby T. Histopathologic features of Burkholderia cepacia pneumonia in patients without cystic fibrosis. Mod Pathol 2000;13:369-72.

21. Bayram M, Babalık M, Bakan ND, Döngel I. Communityacquired Burkholderia cepacia pneumonia: a report of two immunocompetent patients. Tuberk Toraks 2011;59:380-3.

22. Wong KT, Puthucheary SD, Vadivelu J. The histopathology of human melioidosis. Histopathology 1995;26:51-5.

23. Karanth SS, Regunath H, Chawla K, Prabhu M. A rare case of community acquired Burkholderia cepacia infection presenting as pyopneumothorax in an immunocompetent individual. Asian Pac J Trop Biomed 2012;2:166-8.

24. Ishizuka T, Oyama T, Sato M, et al. Fatal pneumonia caused by Burkholderia cepacia 9 months after resection of aspergilloma. Respirology 2003;8:401-3.

25. Kumar S, Dikshit M. Metabolic insight of neutrophils in health and disease. Front immunol 2019;10:2099.

26. Pujol M, Corbella X, Carratala J, Gudiol F. Communityacquired bacteremic Pseudomonas cepacia pneumonia in an immunocompetent host. Clin Infect Dis 1992;15:887-8.

27. Waterer GW, Jones CB, Wunderink RG. Bacteremic community-acquired pneumonia in an immunocompetent adult due to Burkholderia cepacia. Chest 1999;116:1842-3.

28. Suresh G, Prakasha RS, Giridhar BH, Prakash SK. Cavity in the lung: a rare case of Burkholderia cepacia infection. Nitte Univ J Health Sci 2013;3:100-1. 